\title{
Factors affecting general practitioner charges and Medicare bulk-billing: results of a survey of Australians
}

caution is
needed when
considering
changes to
GP fees and
Medicare rebates
because of the
many possible
paths by which
patients' access
to services could
be affected

Richard De Abreu Lourenco

$\mathrm{MEc}$ (Hons)

Patricia Kenny

Marion R Haas

PhD

Jane P Hall

PhD

University of Technology Sydney, Sydney, NSW.

richard.deabreulourenco @chere.uts.edu.au

doi: 10.5694/mjal4.00697
$\mathrm{D}$ ata from Medicare Australia show that $76.9 \%$ of all Medicare Benefits Schedule (MBS) services were bulk billed (charged directly to the Commonwealth without a patient copayment) in the December quarter of 2013. ${ }^{1}$ The proportion was $81.9 \%$ for general practitioner services. ${ }^{1}$ These historically high rates of direct-to-government charges have in part led to calls for the introduction of minimum patient copayments for GP services. While much has been written in the media about the potential impact of such copayments, perhaps less is understood about the factors affecting decisions to bulk bill or to charge patient copayments, and how these factors are linked to patientreported characteristics of general practices.

Studies of bulk-billing have largely focused on GP-specific factors, using data from surveys or large administrative datasets to explore bulk-billing behaviour. Studies of the impact of GP density (number of GPs in a given area) on bulk-billing behaviour have shown that the greater the number of GPs, the greater the propensity to bulk bill., ${ }^{2,3}$ Some surveys of GPs have specifically explored factors determining bulk-billing. One study identified that among a sample of GPs practising in New South Wales, the odds of bulk-billing were higher for those in major cities compared with those in rural areas, for overseas-trained doctors compared with locally trained doctors, and for those with a higher caseload. ${ }^{4}$ Patient income level has also been cited as a factor influencing GPs' bulk-billing decisions. ${ }^{3,5}$

Surveys of patients' experiences of making an appointment with and being treated by a GP have also been done. ${ }^{6-8}$ However, to our knowledge, no Australian survey has captured general practice service-related factors together with detailed information about the personal and health

\section{Abstract}

Objective: To identify factors affecting bulk-billing by general practitioners in Australia.

Design, participants and setting: A community-based survey was administered to Australians aged 16 years or older in July 2013 via an online panel. Survey questions focused on patient characteristics, visit characteristics, practice characteristics.

Main outcome measures: Factors associated with GP bulk-billing.

Results: 2477 respondents completed the survey, of whom 2064 (83.33\%) reported that the practice that they went to for their most recent GP visit bulk billed some or all patients. Overall, 1763 respondents $(71.17 \%)$ reported that their most recent GP visit was bulk billed. Taking into account the duration of visits and the corresponding Medicare Benefits Schedule rebate, the mean out-of-pocket cost for those who were not bulk billed was \$34.09. Results of a multivariate logistic regression analysis suggest that the odds of being bulk billed was negatively associated with larger practice size, respondents having had an appointment for their visit, higher household income and inner or outer regional area of residence. It was positively associated with the presence of a chronic disease, being a concession card holder and having private health insurance. There was no association between bulk-billing and duration of GP visit, age or sex.

Conclusions: Our results indicate that there are associations between patient characteristics and bulk-billing, and between general practice characteristics and bulk-billing. This suggests that caution is needed when considering changes to GP fees and Medicare rebates because of the many possible paths by which patients' access to services could be affected. Our results do not support the view that bulk-billing is associated with shorter consultation times.

characteristics of patients to enable analysis of associations between GP charging behaviour and the characteristics of patients, visits and practices.

We surveyed Australians on recent experiences when visiting a GP to investigate the extent to which bulkbilling is explained by patient characteristics, visit characteristics and practice characteristics. The ability to combine information about respondents with information on the types of primary care services they use (albeit as recalled by patients) offers a new source of data on patient-GP interactions.

\section{Methods}

Our survey was administered to the Pureprofile online panel (http://www.pureprofile.com/au) in July 2013 using the Qualtrics platform (http://www.qualtrics.com). Australians aged 16 years or older were invited to participate via an invitation on their member's home page.

Members of this panel are reimbursed for survey completion according to the time required to complete the survey. The invitation to complete our survey stated that the survey would take up to 15 minutes to complete and that those who completed the survey would be reimbursed $\$ 4.00$.

Respondents were asked about their most recent visit to a GP. The questions focused on: their perceptions of the general practice structure; whether they were bulk billed for the visit, and the fee paid if not bulk billed; their use of primary health 
care services; and demographic details.

Data were analysed using STATA version 12 (StataCorp LP) and conducted using the robust standard errors command to account for the survey nature of the data.

Where a respondent reported paying a fee for their most recent visit, this was compared with the MBS fee for that visit type (for the reported visit duration) to derive a net out-ofpocket cost.

Initial analyses considered frequencies of and correlations between variables thought to be associated with bulk-billing. Associations between these factors and the dependent variable (whether or not the respondent was bulk billed at their most recent GP visit) were first tested using univariate analyses.

Factors for which there was a significant odds ratio (OR) (ie, the $95 \% \mathrm{CI}$ excluded the value 1) were included in a multivariate logistic regression analysis. This type of analysis produces results that can be interpreted as the odds of respondents with a given characteristic, or respondents visiting practices with a certain characteristic, being bulk billed compared with those for whom the characteristic is absent. For parsimony, only results of the multivariate logistic regression analysis are presented in this article.

The respondent factors tested for association were: presence of chronic disease (yes or no); annual household income (low, \$0-\$39999; medium, \$40 000-\$79 999; high, \$80000$\$ 149999$; very high, >\$150000; or unknown); use of any form of government concession card other than a Medicare card (yes or no); having private health insurance (yes, no or unknown); age; region of residence (major city, inner regional, outer regional, remote, or unknown); sex (female or male); and duration of visit (<5 min, 5-19 $\mathrm{min}, 20-39 \mathrm{~min}$ or $>40$ $\min )$.

The practice characteristics tested for association were: the number of GPs in the practice (one or two, more than two, or unknown); and whether the

1 Characteristics of a sample of patients who completed a survey on their most recent visit to a general practitioner and of the Australian adult population $(n=2477)$

\begin{tabular}{|c|c|c|}
\hline & $\begin{array}{c}\text { Sample, number } \\
(\%)\end{array}$ & $\begin{array}{l}\text { Australian adult } \\
\text { population, \%* }\end{array}$ \\
\hline Chronic disease & $1488(60.07 \%)$ & $45 \%$ \\
\hline \multicolumn{3}{|l|}{ GP visits in past year } \\
\hline O or 1 & $557(22.49 \%)$ & $32 \%$ \\
\hline 2 or 3 & $1022(41.26 \%)$ & $31 \%$ \\
\hline $4-11$ & $748(30.20 \%)$ & $27 \%$ \\
\hline 12 or more & $150(6.06 \%)$ & $10 \%$ \\
\hline Female & 1291 (52.12\%) & $51 \%$ \\
\hline \multicolumn{3}{|l|}{ Age, years } \\
\hline $16-24$ & $134(5.41 \%)$ & $17 \%$ \\
\hline $25-34$ & $540(21.80 \%)$ & $17 \%$ \\
\hline $35-44$ & $520(20.99 \%)$ & $18 \%$ \\
\hline $45-54$ & $509(20.55 \%)$ & $17 \%$ \\
\hline $55-64$ & $421(17.00 \%)$ & $14 \%$ \\
\hline $65-74$ & $296(11.95 \%)$ & $9 \%$ \\
\hline 75 or more & $51(2.06 \%)$ & $8 \%$ \\
\hline Unknown & $6(0.24 \%)$ & \\
\hline \multicolumn{3}{|l|}{ Region of residence } \\
\hline Major city & $1824(73.64 \%)$ & $63 \%$ \\
\hline Inner regional & 365 (14.74\%) & $20 \%$ \\
\hline Outer regional or remote & 168 (6.78\%) & $17 \%$ \\
\hline Unknown & $120(4.84 \%)$ & \\
\hline \multicolumn{3}{|l|}{ Private health insurance } \\
\hline Yes & 525 (21.19\%) & $55 \%$ \\
\hline No & 1732 (69.92\%) & \\
\hline Unknown & $220(8.88 \%)$ & \\
\hline \multicolumn{3}{|c|}{$\begin{array}{l}\text { * Data on chronic diseases, GP visits and private health insurance were obtained from the Patien } \\
\text { Experience Survey; }{ }^{;} \text {data on sex and age were obtained from the Census; }{ }^{9} \text { and data on region of } \\
\text { residence were obtained from the Australian Health Survey. }{ }^{6}\end{array}$} \\
\hline
\end{tabular}

respondent had an appointment for the GP visit (yes or no).

As the survey was anonymous, it was not possible to retrospectively collect information from respondents who did not provide it at the time of survey completion. Missing responses were therefore categorised as "unknown".

The study was part of a research program approved by the University of Technology Sydney Human Research Ethics Committee.

\section{Results}

The survey was completed by 2477 individuals. Their characteristics are shown in Box 1 together with those for the Australian adult population. The respondents were comparable to the Australian population with respect to sex and income (median weekly household income for Australia is $\$ 1234^{\circ}$, and the median weekly household income category reported by respondents was \$1150-\$1529). The youngest and oldest age groups were underrepresented in the survey compared with the Australian population and the proportion of respondents living in major cities was higher compared with that for Australian Health Survey participants. ${ }^{6}$

Respondents were in poorer health compared with those in the Patient Experience Survey ${ }^{8}$ in terms of the proportion who reported having a chronic disease and reported numbers of GP visits in the past year. Nearly two-thirds of respondents (1579/2477; 63.75\%) reported going 
to the GP three or fewer times in the past year.

Most visits (1888/2477; 76.22\%) lasted 5-19 minutes (consistent with a level B consultation). A lower proportion of respondents reported having private health insurance cover compared with those in the Patient Experience Survey.

Most respondents reported that they had a usual general practice $(2222 / 2477 ; 89.71 \%)$ and that they usually saw the same GP in the practice that they went to most often (1989/2477; 80.30\%).

The survey question on bulk-billing referred to the most recent GP visit, regardless of whether that visit was with the respondent's usual GP. Most respondents (2064/2477; 83.33\%) reported that the practice they went to for their most recent visit bulk billed some or all patients. A majority of respondents $(1763 / 2477 ; 71.17 \%)$ reported that their most recent GP visit was bulk billed, and the remaining 714 provided information about fees paid at their most recent visit.

Of those who were not bulk billed, the mean fee charged was \$64.04. Taking into account durations of visits and corresponding MBS rebates, the mean out-of-pocket cost was estimated to be \$34.09. These values exclude 189 respondents who reported being charged a fee less than the MBS fee associated with their visit duration (ie, those for whom an out-of-pocket cost estimate could not be calculated).

Of those who were not bulk billed, $39.92 \%$ (285/714) had an annual household income of less than $\$ 80000$. Of those who were bulk billed, $53.37 \%$ (941/1763) had an annual household income of less than $\$ 80000$.

The univariate analyses showed that all factors other than sex and duration of visit were associated with bulkbilling. Results of the multivariate logistic regression analysis, containing the remaining factors, are shown in Box 2. An OR of 1 or close to it indicates no association between a given factor and the odds of bulk-billing. Factors for which the 95\% CI does not include the value 1 are statistically significant $(P<0.05)$.

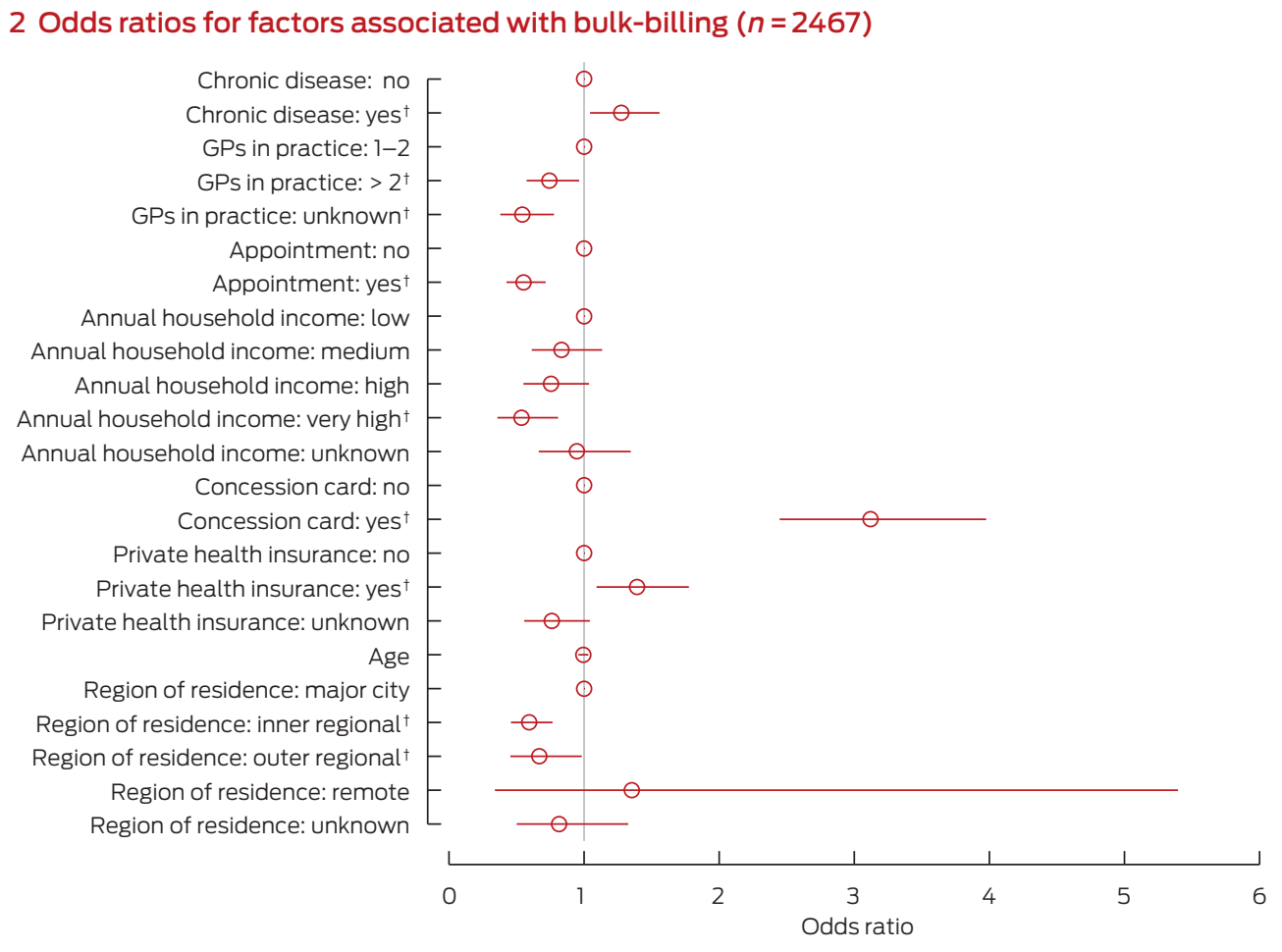

* Multivariate regression statistic Wald $\chi^{2}=222.68(P<0.001)$. Bars represent $95 \% \mathrm{Cls}$. For each category except age, the base factor appears without a $95 \% \mathrm{Cl}$ and straddles the line at 1 . The number of respondents included was reduced by 10 due to six missing observations for age and four missing observations for concession card status. $\uparrow P<0.05$ compared with base factor.

These results show that there are higher odds of being bulk billed among patients with chronic diseases (OR, 1.28; 95\% CI, 1.04-1.56), those with a concession card (OR, 3.12; 95\% CI, 2.45-3.98) and those with private health insurance (OR, 1.39; 95\% CI, 1.09-1.78). However, the odds of being bulk billed are lower for those on very high incomes compared with those on low incomes (OR, 0.54; 95\% CI, 0.36-0.81) and for those living in inner and outer regional areas compared with major cities (OR, 0.59; 95\% CI, 0.46-0.77 and OR, 0.67; 95\% CI, $0.45-0.98$, respectively).

Service-related characteristics were also important in explaining bulkbilling behaviour. Respondents with an appointment for their last visit had a lower odds of being bulk billed compared with those without an appointment (OR, 0.55; 95\% CI, 0.43-0.72). Similarly, respondents who visited practices that had more than two GPs and those who reported not knowing how many practitioners were in a practice, had a lower odds of being bulk billed than those who attended a practice with one or two
GPs (OR, 0.74; 95\% CI, 0.57-0.96 and OR, 0.54; 95\% CI, 0.38-0.78, respectively). The results of separate regressions including sex and duration of visit showed that neither of these factors was significant and did not add to the explanatory power of the analysis (data not shown).

\section{Discussion}

Our results indicate that while a high proportion of GP visits are bulk billed, nearly one-third of respondents paid a fee at their most recent visit. For some respondents, introduction of additional copayments would therefore not be a significant departure from the status quo. However, additional copayments would be novel for many patients and our analysis suggests that these could cause difficulties for a substantial proportion of those individuals.

We found a higher propensity to bulk bill individuals with lower income levels, those with chronic diseases and those with concession cards. These are the groups who would be 
the most disadvantaged by the introduction of additional copayments for GP visits.

Perhaps more surprising was the finding that having private health insurance was positively linked with being bulk billed, after adjusting for income and presence of a chronic disease. In a recent study, it was observed that Australian holders of private health insurance were more likely to be healthier than those without insurance. ${ }^{10}$

We speculate that healthier individuals might be more willing to discriminate between GPs on the basis of bulk-billing and better able to find bulk-billing practices. A more direct relationship between private insurance and bulk-billing is expected to emerge if a pilot program currently underway by IPN (Independent Practitioner Network) and Medibank Private to guarantee bulk-billing of GP appointments for Medibank members proves to be successful. ${ }^{11}$

Respondent region of residence was also associated with bulk-billing, with lower rates among residents of inner and outer regional areas despite higher bulk-billing incentive payments for GPs in regional, rural and remote areas. This is consistent with the results of a previous study in which an almost sevenfold higher odds of bulk-billing among GPs in metropolitan areas was observed compared with rural areas. ${ }^{4}$ This could reflect GP density rather than respondent-related factors; GP concentrations in major cities lead to greater price competition between practices for respondents, which results in an increased likelihood of bulk-billing. ${ }^{2,3}$ This diminishes as the concentration of practices reduces in less densely populated areas.
As well as respondent-specific factors, we analysed factors relating to visits and practices. Having an appointment was found to halve the odds of being bulk billed compared with not having an appointment. This might indicate that practices which are able to accept non-urgent "walk-ins" (owing to flexible schedules or availability of practitioners) have spare capacity hence are more likely to bulk bill to encourage demand. Alternatively, practices might discriminate and select patients who are prepared to pay by offering reduced waiting times. This requires further investigation at the general practice level.

Similarly, the impact of practice size on bulk-billing behaviour warrants closer investigation. Our results indicate that smaller practices (one or two practitioners) had a higher odds of bulk-billing than those with more practitioners and those in which the number of practitioners was not known by respondents (assuming that most respondents would be able to recall if a practice had one or two practitioners only, the latter category could be grouped with the "two or more" group). In practices with one or two practitioners, there may be less capacity to compete on the basis of service offerings (eg, multiple practitioners, co-located pathology services) and amenities, resulting in greater price competition. For these practices, increased rates of bulk-billing might be a key point of differentiation from other practices. This would reinforce the notion that practice structure, even allowing for the potential effects of flexible arrangements, is a determinant of billing practices.

A limitation of our study is that the sample differed in terms of chronic diseases, numbers of GP visits in the past year, age, region of residence and private health insurance status. The sample was representative in terms of income and sex distributions, but it is possible that there were other unmeasured differences related to self-selection into the online panel. Despite the differences, the large sample size meant that there were sufficient numbers within the relevant subgroups to provide the power to detect differences in the likelihood of being bulk billed, while controlling for effects of the remaining population characteristics.

We combined respondent-specific factors with respondent-reported practice characteristics to investigate demand and supply influences on bulk-billing. Both are important since changes in patient factors (eg, ability to pay for care) and GP factors (supply of care) influence the use of primary health care services. ${ }^{12}$ As expected, people with chronic diseases and those with low household incomes were less likely to be charged. We also found several interesting associations that warrant further research, such as that between health insurance status and bulk-billing, and that between general practice structure and bulk-billing. Nonetheless, our findings are relevant when considering potential changes to Medicare funding that might affect bulk-billing by GPs, which will affect individuals' capacity to access services.

Acknowledgements: This research was conducted at the Centre for Research Excellence in the Finance and Economics of Primary Care (REFinE-PHC), which is supported by a grant from the Australian Government Department of Health.

Competing interests: No relevant disclosures. Received 13 May 2014, accepted 28 Aug 2014. 
1 Department of Health. Quarterly medicare statistics. http://health.gov.au/internet/main/ publishing.nsf/Content/Quarterly-MedicareStatistics (accessed Apr 2014).

2 Hopkins S, Speed N. The decline in 'free' general practitioner care in Australia: reasons and repercussions. Health Policy 2005; 73: 316-329.

3 Savage E, Jones G. An analysis of the General Practice Access Scheme on GP incomes, bulk billing and consumer copayments. Aust Econ Rev 2004; 37: 31-40. doi: 10.1111/j.1467-8 462.2004.00306.x.

4 Khan A, Hussain R, Plummer D, Minichiello V. Factors associated with bulk billing: experience from a general practitoners' survey in New South Wales. Aust N Z J Public Health 2004; 28 : 135-139.
5 Jones G, Savage E, Hall J. Pricing of general practice in Australia: some recent proposals to reform Medicare. J Health Serv Res Policy 2004; 9 Suppl 2: 63-68.

6 Australian Bureau of Statistics. Australian health survey: first results, 2011-12. Canberra: ABS, 2012. (ABS Cat. No. 4364.0.55.001.) http://www.abs.gov.au/AUSSTATS/abs@. nsf/DetailsPage/4364.0.55.001201112?OpenDocument (accessed Jan 2014).

7 Schoen C, Osborn R, Squires D, Doty MM. Access, affordability, and insurance complexity are often worse in the United States compared to ten other countries. Health Aff (Millwood) 2013; 32: 2205-2215.

8 Australian Bureau of Statistics. Patient experiences in Australia: summary of findings, 2012-13. Canberra: ABS, 2013. (ABS Cat. No. 4839.0.) http://www.abs.gov.au/ausstats/ abs@.nsf/mf/4839.0 (accessed Jan 2014).
9 Australian Bureau of Statistics. 2011 Census QuickStats. http://www.censusdata.abs.gov. au/census_services/getproduct/census/2011/ quickstat/0?opendocument\&navpos=220 (accessed Jan 2014).

10 Doiron D, Jones G, Savage E. Healthy, wealthy and insured? The role of self-assessed health in the demand for private health insurance. Health Econ 2008; 17: 317-334.

1 Medibank. Medibank and IPN trial to give members more options to access to GPs at no cost [media release]. 10 Jan 2014. http://www. medibank.com.au/About-Us/Media-CentreDetails.aspx?news=535 (accessed May 2014).

12 Day SE, Alford K, Dunt D, et al. Strengthening Medicare: will increasing the bulk-billing rate and supply of general practitioners increase access to Medicare-funded general practitioner services and does rurality matter? Aust New Zealand Health Policy 2005; 2: 18. 